\title{
Localization and Organization of the Central Pattern Generator for Hindlimb Locomotion in Newborn Rat
}

\author{
Jean-René Cazalets, ${ }^{1}$ Michel Borde, ${ }^{2}$ and François Clarac ${ }^{1}$ \\ ${ }^{1}$ CNRS, NBM, BP 71, 13402 Marseille Cedex 20, France and 'Laboratorio de Neurofisiologia, Facultad de \\ Medicina, 11800 Montevideo, Uruguay
}

\begin{abstract}
An in vitro preparation of newborn rat isolated brainstem/ spinal cord was used in order to locate the spinal network responsible in mammals for producing patterned locomotor activity. The spinal cord was partitioned by building Vaseline walls at various lumbar levels. When a mixture of serotonin and $\mathrm{N}$-methyl-D,L-aspartate was bath applied to the upper lumbar cord (L1/L2 segments), rhythmic locomotor-like activity was induced and recorded in all the lumbar segments (from L1 to L5). Conversely, when the mixture of transmitters was bath applied to the lower lumbar cord, only tonic activity was induced in the lower lumbar segments. Intracellular recordings performed on motoneurons revealed that during elicited L1/L2 locomotor-like activity, they received a rhythmic synaptic drive that was often below the threshold for spiking, because the excitability of the neurons was too low. When the L1/L2 segments were isolated, their burst production capacities remained. The network located at the L1/L2 level was found to be responsible not only for generating the rhythm but also for organizing its alternating pattern. We demonstrated that the rhythmic synaptic drive that the motoneurons receive during locomotor-like activity comes directly from the L1/ L2 network and that there is no relay at the segmental level. We conclude from our study that the network that organizes locomotion in the newborn rat is not segmentally distributed but is restricted to a specific part of the cord. This finding has important consequences, since it means that it is now feasible to study the activity of the rhythmic spinal network independently from that of the motoneurons.
\end{abstract}

[Key words: electrophysiology, spinal cord, locomotion, central pattern generator, motoneuron drive, Intersegmental coordination]

Like other rhythmic behaviors (for review, see Delcomyn, 1980; Grillner, 1985; Cohen et al., 1988), locomotor activity is centrally generated by neuronal networks called central pattern generators (CPGs). The best way of demonstrating the existence of such networks is to directly remove the nervous system from the animal and record the activity in these isolated conditions. Although this has been achieved in many preparations from in-

Received Aug. 25, 1994; revised Feb. 1, 1995; accepted Feb. 3, 1995

This work was supported by a grant from NATO and by INSERM Grant 920803. Wo are indebted to John Alexander Simmers and Richard Miles for their help and encouragements and to Jon Barnes for help in final English version. Many thanks to $R$. Navarro for caring for the pups.

Correspondence should be addressed to Cazalets Jean-René, CNRS, NBM, 31 chemin Juseph Aiguier, BP 71, 13402 Marseille Cedex 20, France.

Copyuight $\odot 1995$ Society for Neuroscience 0270-6474/95/154943-09\$05.00/0 vertcbratcs (Kristan and Calabrese, 1976; Sillar and Skorupski, 1986; Chrachri and Clarac, 1990) and lower vertebrates (Cohen and Wallén, 1978; Grillner et al., 1981; Roberts et al., 1983), locomotor-like activity was only quite recently recorded in mammals in an in vitro isolated spinal cord preparation (Kudo and Yamada, 1987 ; Smith et al., 1987; Cazalets et al., 1990, 1992). The exact location of the CPG itself is still unknown in mammals, and to date it has not been possible to tell whether the basic oscillator occupies a specific location in the spinal cord or whether it presents a multisegmental distribution in the lower spinal cord. The data collected on lower vertebrates (Xenopus, lamprey) might not be suitable for answering this question, since the locomotor behavior studied is swimming activity resulting from the longitudinal transmission of a metachronal wave, which is transmitted through a multisegmented system (Kahn and Roberts, 1982; Cohen, 1987; Grillner and Matsushima, 1991; Tunstall and Sillar, 1993). Most of the experiments performed on mammals in order to locate the rhythmic network have involved the use of markers of neuronal activity such as deoxyglucose (Viala et al., 1988), the C-Fos labeling of neurons (Barajon et al., 1992) or the fluorescent probe sulforhodamine (Kjaerulff et al., 1994). Although these methods have provided some general information about the pattern of activity in the spinal cord, there exists no means of preventing many celss, including cells that are not directly participating in the rhythm generation, from being labeled. On the other hand, on the basis of experiments using transection or cooling techniques, it has been concluded that, in the cat, the bursting capacity is distributed along the spinal cord (Deliagina et al., 1983). Here, we approached this problem using an isolated newborn rat spinal cord preparation to which various techniques were applied. In the first step, we built Vaseline walls at various levels along the lumbar cord in order to determine the minimal portion of spinal cord that was required for burst generation. The advantage of this method is that there is no damage to the spinal cord and that one can separately control the extracellular medium of the various compartments. We conclude that, in the rat, the spinal network producing rhythmic activity is located between segments T13 and L2, while the lower lumbar segments, which contain most of the motoneurons innervating the hindlimbs, to not participate at all in the rhythm genesis.

\section{Materials and Methods}

Experiments $(n=29)$ were performed on Wistar rats aged $1-4$ d raised at our laboratory. The pups were deeply anesthetized with ether, prior to decapitation. The skin was then removed and the vertebrae were cut dorsally. The spinal cord was removed and placed in the recording chamber (Fig. 1A). The isolated brainstem/spinal cord was continuously superfused with an oxygenated $\left(95 \% \mathrm{O}_{2} / 5 \% \mathrm{CO}_{2}\right)$ physiological saline (composition in mM: $\mathrm{NaCl}, 129 ; \mathrm{KCl}, 4 ; \mathrm{CaCl}_{2}, 2.5 ; \mathrm{MgCl}_{2}, 1,14$; 


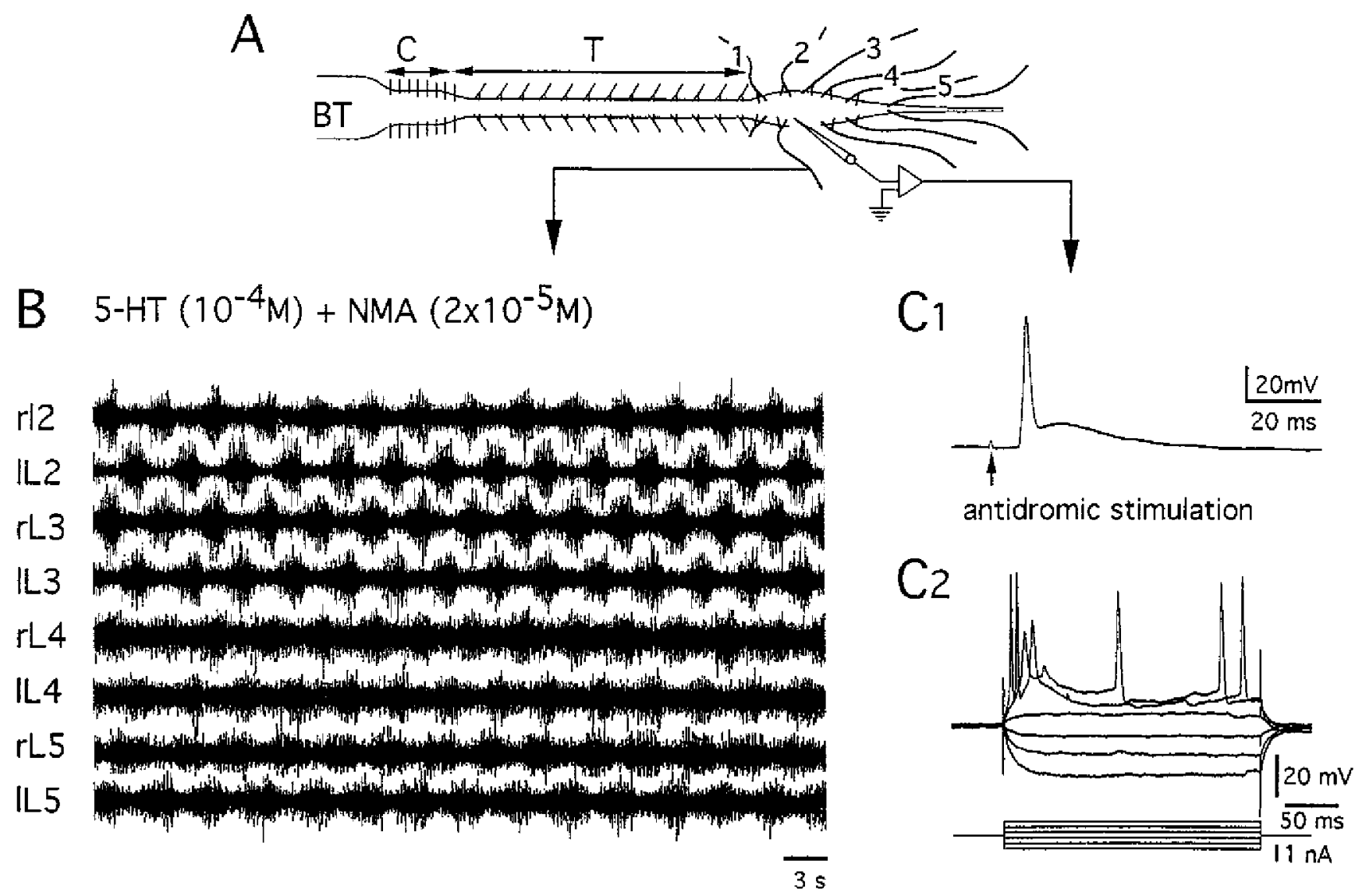

Figure 1. Recording of locomotor-like activity in the isolated brainstem/spinal cord preparation. $A$. Diagram of the isolated spinal cord. $B$, Simultaneous extracellular recordings of four pairs of lumbar ventral roots. Locomotor-like activity was induced by bath application of 5-HT/NMA. $C$, Intracellular recording of a motoneuron. Electrical stimulation of left ventral root 5 evoked an antidromic action potential (C/), Current-voltage relationship of a motoneuron (C2). The threshold for spiking was between 0.5 and $1.5 \mathrm{nA}$. $r L$ and $l L$, right and left lumbar ventral roots $2-5$.

$\mathrm{NaH}_{2} \mathrm{PO}_{4}, 0.5 ; \mathrm{NaHCO}_{3}, 25$; glucose, 10 ), adjusted to $\mathrm{pH}=7.4$ with $\mathrm{HCl}$. The bath temperature was kept constant at $25^{\circ} \mathrm{C}$. For details of the dissection procedure, stage,-and thermoregulation system, see SqalliHoussaini et al. (1991) as well as Cazalets et al. (1992, 1994).

Extracellular recordings. The motor output from the ventral roots was recorded using extracellular platinum pin electrodes insulated with Vaseline. The signals were amplified, displayed, and stored using classical electrophysiological devices. Locomotor-like activity was induced by bath applying a mixture of serotonin $\left(5-\mathrm{HT}, 10^{-4} \mathrm{M}\right)$ and $N$-methylD,L-aspartate (NMA, $2 \times 10^{-5} \mathrm{M}$; Fig. $1 B$; Sqalli-Houssaini et al., 1991 , 1993). Figure $1 B$ shows the motor pattern elicited when the neuroactive compounds were bath applied to the whole spinal cord. It consists of right and left alternating bursts of action potentials, which were recorded in lumbar ventral roots $1-5$ (L1 to L5). The identification of the lumbar segments was performed in several ways. They could be identified by counting them from cervical 1 . This was not systematically done, however, since we found that the ventrat root L5 could be clearly identified as the most caudal larger root at the lumbar level. This anatomical criterion was correlated with a functional one, since the activity recorded in this root was always in antiphase with that recorded in the ipsilateral ventral root $\mathrm{L} 2$, as determined in more than 40 experiments in which each pair of ventral root L2, L3, and L5 were simultaneously recorded during sequences of stable locomotor-like activity.

The pia was removed to perform intracellular recordings. Glass microelectrodes were pulled using a Sutter PC-80 micropipette puller and filled with $\mathrm{K}$ acetate $2 \mathrm{M}(40-60 \mathrm{M} \Omega$ ). KCl $3 \mathrm{~m}$ filled electrodes (30$40 \mathrm{M} \Omega$ ) were also occasionally used in order to detect more clearly the synaptic drive to the motoneurons (this will be specified in the text). Intracellular potentials were amplified through an Axoclamp $2 B$ amplifier (Axon Instruments, Foster City, CA). Stable intracellular recordings (for up to $6 \mathrm{hr}$ ) could be thus obtained. Only the neurons with at least a $-60 \mathrm{mV}$ resting membrane potential and an antidromic potential of at least $70 \mathrm{mV}$ were considered for analysis. Data were collected using an Instrutech ITC-16 interface connected to Macintosh Quadra 650. The software programs for data acquisition and analysis were from Axom Instrument. The motoneurons were identified by recording their antidromic potential in response to ventral root stimulation (Fig. 1C). The mono- and polysynaptic EPSPs evoked in the motoneuron in response to dorsal root stimulation were used to test the efficiency of the various blockers that we used (see text for details).

Partitioning of the lumbar spinal cord. In order to determine which segment of the lumbar spinal cord was capable of generating rhythmic activity, we built Vaseline walls at various levels. Vaseline was contained in a glass syringe and ejected through a thin needle $(0.5 \mathrm{~mm})$, The wall thickness was around two-thirds of a segment. The water tightness of the wall was checked by filling the pool until a meniscus was created. If no changes in the saline level occurred after several minutes, the wall was taken to be watertight. The location of the barriers will be specified in each case in the Results section. We performed experiments to evaluate the extent of diffusion throughout the tissue. A barrier was made at the level of a root. Methylene blue $\left(10^{-3} \mathrm{M}\right)$ dissolved in saline was perfused on one side. The dye was applied under the same conditions as a saline containing drug before fixing the cord in paraformaldehyde $(4 \%$ in phosphate buffer $0.1 \mathrm{M})$. This procedure showed that no visually detectable diffusion of the dye occurred beyond the inner side of the wall that was in contact with the methylene blue containing saline. The inner side of the wall was therefore taken as the location reference when establishing the limits of the various parts of the spinal cord.

Modified salines. The drugs were prepared at $10^{-2} \mathrm{M}$ in saline, frozen, and stored at $-20^{\circ} \mathrm{C}$. They were generally dissolved in the saline at the appropriate concentration prior to use. Under our superfusing conditions, the effects of the drugs began to take place $5 \mathrm{~min}$ after the drug reached the Petri dish (the time required for total replacement of the normal saline and diffusion through the tissue). The drug was bath applied until a steady state was reached and measurements could be 

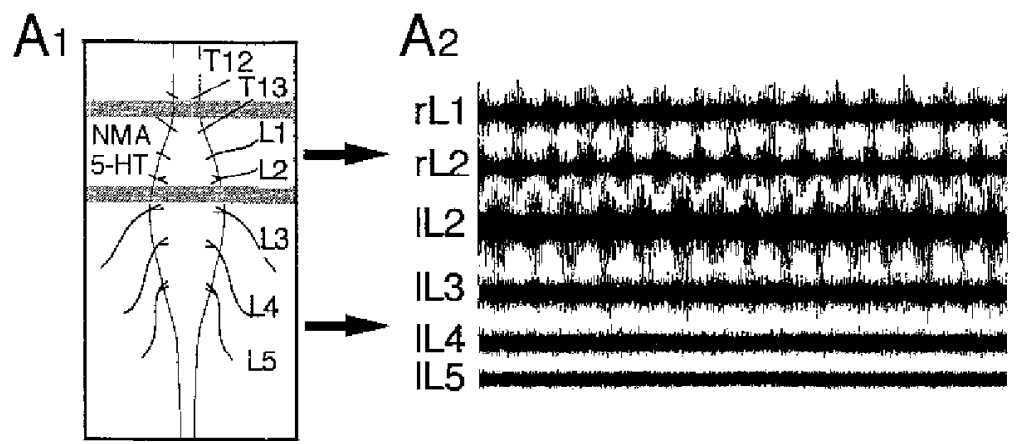

$\mathrm{A}_{3}$

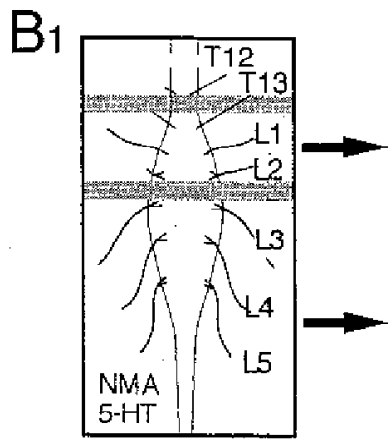

$\mathrm{B} 2$

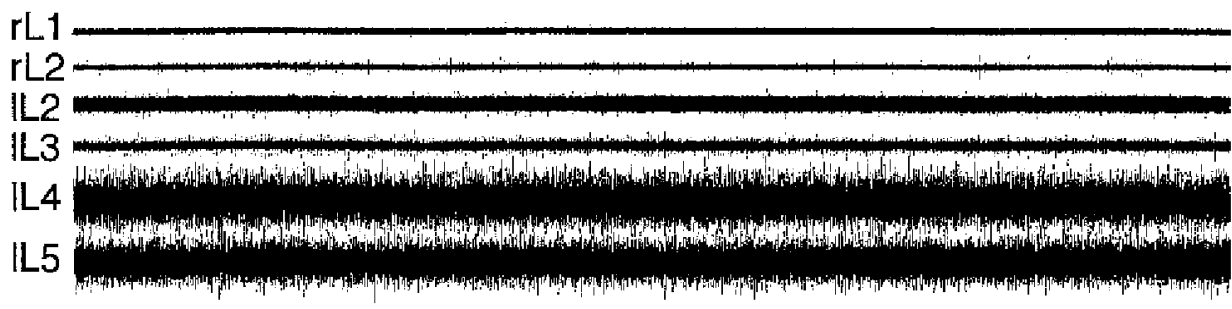

$5 \mathrm{~s}$

Figure 2, Rhythmic activity arising in spinal segments. $A /$ and $B /$, Diagrams of experimental preparation. $A$, When a mixture of 5-HT and NMA was bath applied to upper lumbar segments (Al), locomotor-like activity was initiated and recorded either in the first lumbar roots (L1 to L3, A2) or in the all the lumbar roots (I.1 to I.5, A3) $B$, When S-HT/NMA were bath applied to the lower lumbar segments (BI, L3 to L5), only tonic activity was elicited in L4 and L5 $(B 2)$. The recordings in $A 2$ and $B 2$ are from the same experiments. Abbreviations; $r L$, right lumbar; $l L$, left lumbar; $T$, thoracic.

performed (Cazalets et al, 1992). We selectively perturbed the neuronal activity by superfusing salines with various ionic compositions. To suppress the polysynaptic pathways, we used a saline enriched with $\mathrm{Ca}^{2+}$ (7.5 mM) and $\mathrm{Mg}^{2+}$ ( $8 \mathrm{~mm}$ ) (Berry and Pentreath, 1976; Jahr and Yoshioka, 1986) or containing $1 \mathrm{~mm}$ mephenesin (Lev-Tov and Pinco, 1992). The efficiency of these modified salines was tested in each experiment on the postsynaptic potentials evoked in motoneurons by dorsal root stimulation. All the drugs were putchased from Sigma (St. Louis, MO).

Measurements. The period was defined as the time between the onset of two bursts of activity. The phase relationship between two ventral roots was defined as the latency at which the onset of a burst occurred during the cycle of the other root, divided by the cycle period (see Cazalets et al., 1990b). The statistical values are given as the mean \pm $\mathrm{SD} ; n$ refers either to the number of cycles or to the number of experiments.

\section{Results}

\section{Location of the network}

In order to explore the bursting capabilities of the various parts of the lumbar spinal cord and to assess their contributions to thythm generation, we built Vaseline walls at various levels to allow exogenous pharmacological treatment to restricted areas of the spinal cord. First, we will consider the bursting capabilities of the midlumbar segments. The first Vaseline wall was built at the T13 level (gray bar Fig. $2 A 1, B I$ ) in order to separate off the upper part of the cord. From one experiment to another, a second wall was placed at various points along the lumbar cord. When it was placed below segment L2 (gray line, Fig. 2; $n=23$ experiments), the bath application of NMA/5-HT induced locomotor-like activity only when superfused into the upper lumbar compartment. Under these conditions, rhythmic discharge was observed either in the upper ventral roots only (L1/ L2; Fig. 2A2) or in all lumbar ventral root (Fig. 2A3). On the other hand, when transmitters were bath applied to the caudal part, in all cases they only induced tonic activity in ventral roots L3 to L5 (Fig. 2B2). In two experiments in which the Vaseline wall was built between $\mathrm{L} 1$ and $\mathrm{L} 2$, a weak, irregular activity was generated when 5-HT/NMA was bath applied in both the rostral and the caudal lumbar pool.

Even when no activity was recorded in the lower lumbar ventral roots (as, e.g, in the case of Fig. 2A2), intracellular recordings showed that the motoneurons consistently received a rhythmic synaptic drive when the 5-HT/NMA was bath applied to segments L1/L2. In the experiment shown in Figure 3, a motoneuron from the left lumbar root number 5 (MN IL5) was tested. Bath application of 5-HT/NMA to the rostral pool (Fig. 3AI) elicited a rhythmic alternating pattern and the motoneuron shythmically fired bursts of action potentials (Fig. 3A2); whereas when 5-HT/NMA was bath applied to the caudal part (Fig. 3BI) of the spinal cord the neuron only fired tonically (Fig, 3B2).

In many cases, no activity was detected in the caudal ventral roots ( $\mathrm{L} 3$ to L5) when the transmitters were bath applied to the rostral pool (see Fig. 2 legend). As shown in Figure 4, this was due to the fact that the excitability of the neurons was too low so that they were below the threshold for spiking. In this experiment, the coxygeal part of the spinal cord was stimulated. Under the control conditions (Fig. 4A), this stimulation (which gives rise to massive sensory inputs) triggered only one burst of activity. In the same experiment, locomotor-like activity was then induced by perfusing the rostral segments with 5-HT/NMA, and this triggered rhythmic alternating bursts of action potentials only in the L2 ventral roots (Fig. $4 B$, left). At the same time, the neuron was rhythmically depolarized in relation with the left L2. The same stimulation as under the control conditions then elicited rhythmic locomotor bursting in all the ventral roots, and 
$A_{1}$

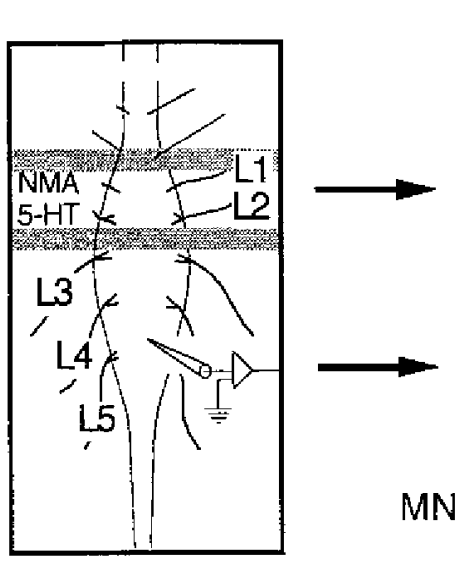

$\mathrm{A} 2$

MN IL5
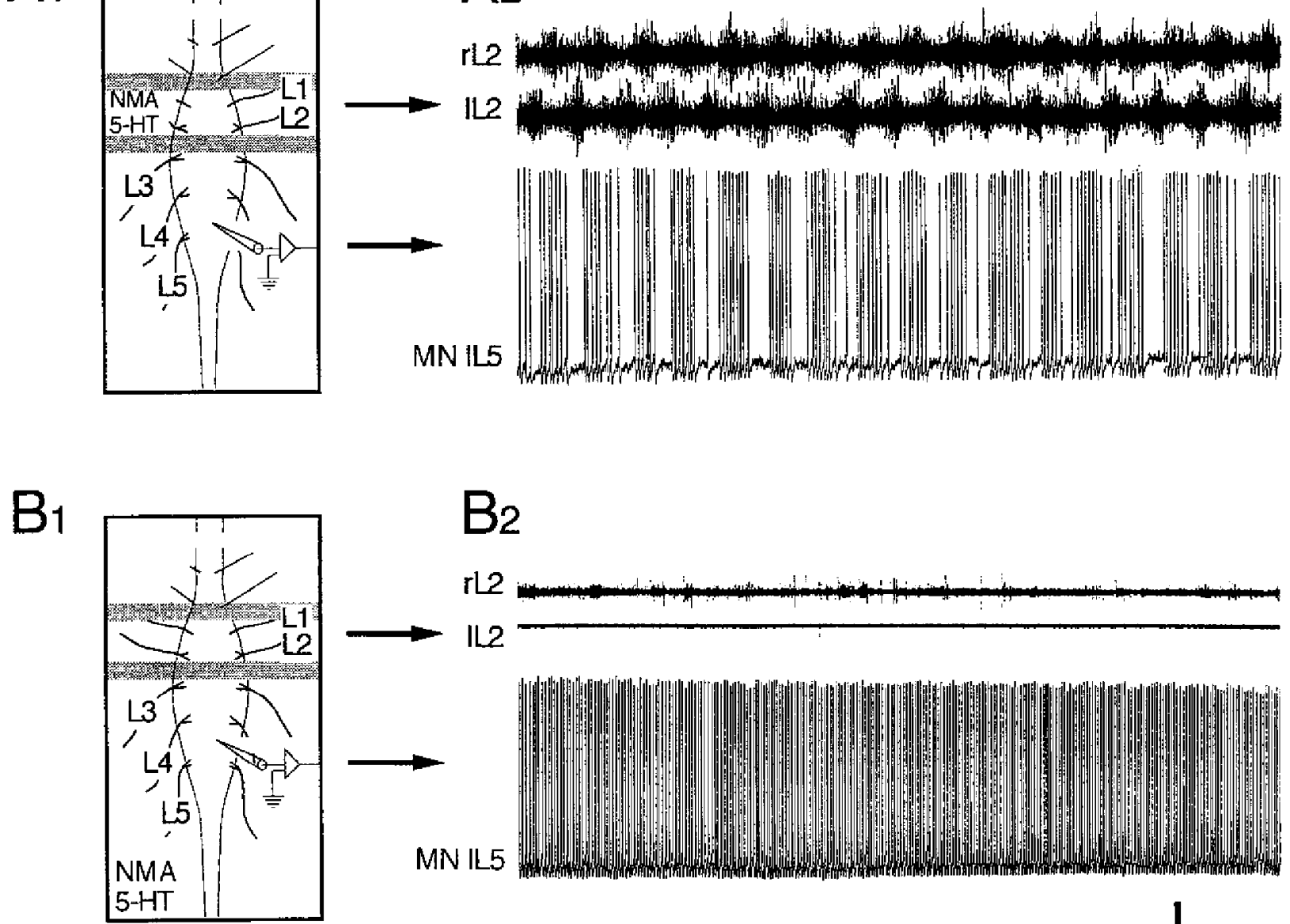

$\mathrm{B}_{2}$

rl2

IL 2

MN IL5

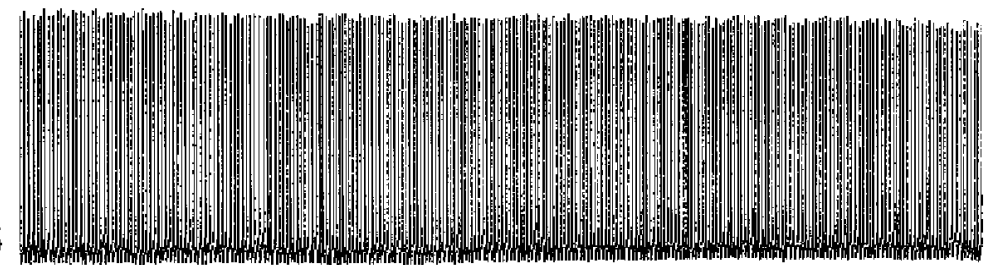

$20 \mathrm{mV}$

$5 s$

Figure 3. During L1/L2 induced locomotion, the motoneurons located in the lower lumbar segments receive a locomotor drive. $A I$ and $B I$, Diagrams of the experimental preparation. 5-HT/NMA were bath applied to upper $(A /)$ or lower $(B I)$ lumbar cord. Intracellular recordings of a motoneuron was performed in L5 while simultaneously recording the ventral root L2. A2, During locomotor-like activity induced in L1/L2 the motoneuron recorded in the left lumbar segment L5 (MNLL5) was rhythmically depolarized and spontaneously fired bursts of action potentials in relation with the burst recorded in IL2. B2, When 5-HT/NMA was bath applied to the lower lumbar cord, the motoneuron only discharged tonically and no activity was recorded in the ventral roots.

the neuron fired action potentials (Fig. $4 B$, right). The depolarization induced by the stimulation thus brought the motoneurons above their fring threshold.

Although the caudal segments (L3 to L6) do not exhibit oscillatory properties under out experimental conditions, it might be argued that they may directly influence the oscillatory activity in T13-L2 via internal loops, and that their presence is necessary for rhythmic activity to be produced in rostral segments. To test this hypothesis, we isolated the L1/L2 segments by suppressing the spiking activity with the fast $\mathrm{Na}^{+}$channel blocker tetrodotoxin (TTX; Fig. 5A1). In Figure 5A, rhythmic activity was extracellularly recorded in $\mathrm{L} 2$ and intracellularly at the $L 5$ level. The bath application of 5-HT/NMA to L1/L2 under control conditions (Fig. 5A2) induced alternating activity and the motoneuron in L5 was rhythmically depolarized. The bath application of TTX to the whole brainstem and spinal cord except the L1/L2 segments (Fig. 5A3) abolished the synaptic drive in the L5 motoneuron, while the activity in L2 persisted. In Figure $5, B$ and $C$ show that there were no changes either in the motor period during TTX bath application (Fig. $5 B$ ) or in the phase relationships (Fig. 5C).

\section{Organization of the network}

One question that arises is whether, in addition to this rhythmic compartment, there may also exist other elements responsible at the segmental level for organizing the alternating right and left and the flexor/extensor pattern. Is the control of the alternation distributed along the spinal cord, or is it organized by a discrete set of neurons that then drives the follower motoneurons?

We first tested that the right and left alternation was not segmentally organized by using preparations in which the spinal cord was hemisected along the midline from the caudal end to the second lumbar segment (Fig. 6A). Under these conditions, the bath application of 5-HT and NMA still induced alternating activity not only in segments $L 1$ and L2 but also in segments L3 to L5 (Fig. 6B). Further evidence was provided by the results obtained using strychnine. This compound, acting on the glycinergic receptors, is known to block the inhibitory connections 

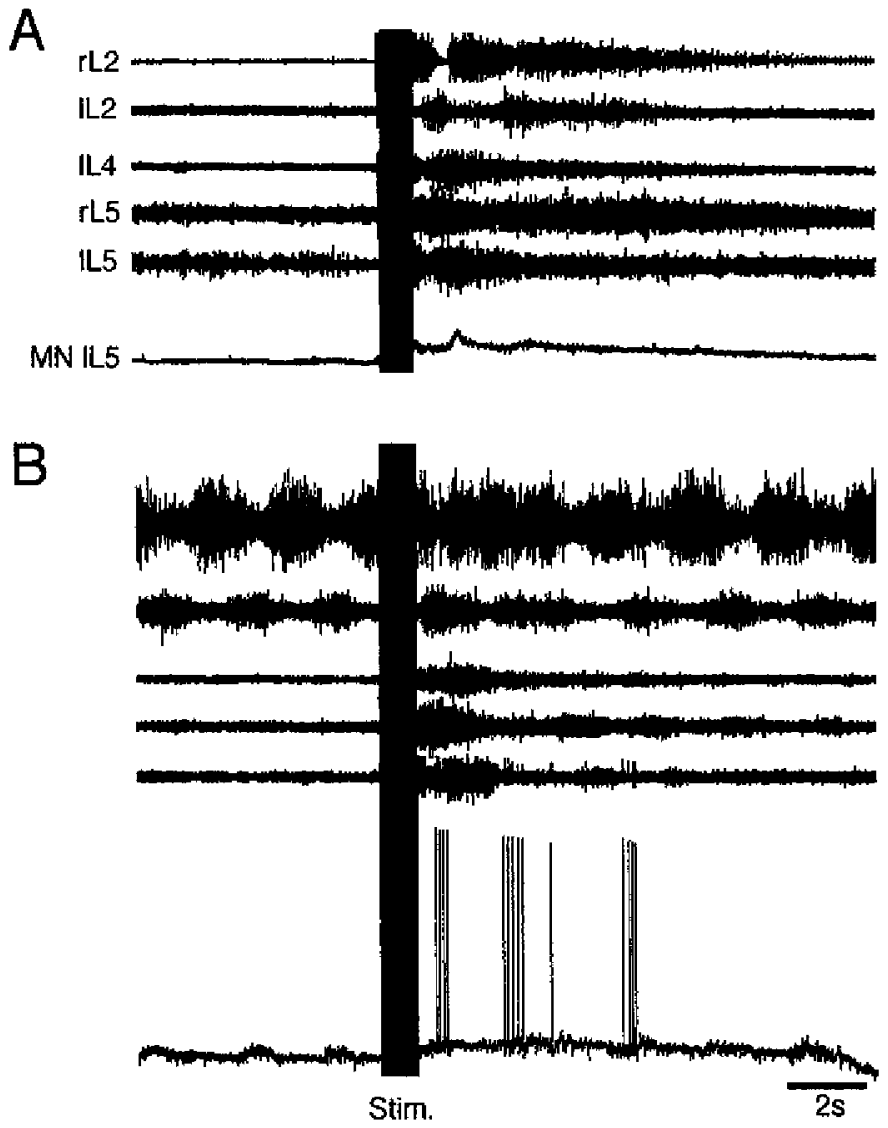

Figure 4. Subthreshold synaptic drive onto motoneurons. A, Under control conditions, electrical stimulation $(50 \mathrm{~Hz}, 1 \mathrm{sec})$ of the coxygeal spinal cord elicited only tonic activity, which outlasted the stimulation. $B$, In the presence of 5-HT/NMA, a rhythmic alternating pattern was initiated in the ventral root L2. No activity was recorded in the lower lumbar roots (L4/L5), although the intracellularly recorded motoneuron was rhythmically depolarized. The same stimulation as under control conditions brought the neurons above their firing threshold and elicited rhythmic bursts of action potentials in all the ventral roots and in the intracellularly recorded motoneuron.

between the right and left sides (Grillner and Wallén, 1980; Cohen and Harris-Warrick, 1984; Soffe, 1987; Kudo et al., 1991). Under our experimental conditions, the bath application of strychnine disrupted the locomotor-like rhythm only when applied to L.1/L.2, while it had no effect on the rhythm when applied to the cord between L3/L5 (data not shown).

Lastly, we suppressed the polysynaptic pathways in the caudal part by perfusing either a saline enriched with $\mathrm{Ca}^{2+}$ and $\mathrm{Mg}^{2+}$ or one containing mephenesin (see Materials and Methods). Figure 7 shows the action of these modified salines on the EPSPS evoked in a motoneuron by dorsal root stimulation. The longlatency EPSPs were completely abolished while the fast rising response was maintained. The same results were obtained with the two salines, although the high $\mathrm{Ca}^{2+} /$ high $\mathrm{Mg}^{2+}$ containing saline was slightly more efficient than the mephenesin containing saline. Figure 8 shows that although polysynaptic pathways were suppressed by the saline containing mephenesin, the synaptic drive onto the motoneurons persisted (Fig. 8BI). In addition, it shows that mephenesin (as well as the high $\mathrm{Ca}^{2} \cdot 1 / \mathrm{High}$ $\mathrm{Mg}^{2 *}$ containing saline) probably acted by decreasing the neuronal excitability, since the motoneuron that fired bursts of action potentials in response to positive current injection under the control condition (Fig. 7A2) no longer discharged in the presence of mephenesin (Fig. 7B2). In order to obtain a maximal blockade, we also bath applied a saline enriched with $\mathrm{Ca}^{3+} / \mathrm{Mg}^{2+}$ containing mephenesin. Even in this case the drive persisted (data not shown). These data therefore demonstrate that the connections between the network in $\mathrm{L} 1 / \mathrm{L} 2$ and the motoneurons are monosynaptic.

\section{Discussion}

We conclude that the network that generates locomotor-like activity in a mammal is restricted to a specific part of the spinal cord. Our experiments also show that the relationships between this network and its target motoneurons in other segments are direct and do not require a polysynaptic pathway.

Two methodological points were critical in this study. The first concerned the extent of diffusion of the neuroactive compounds used to activate the CPG through the Vaseline wall. As already mentioned in the Materials and Methods section, the water tightness was tested at the beginning of the experiment. Although it could be easily established that there were no leakage, it was more difficult to test the degree of diffusion through the tissue. As described above (see Materials and Methods), it was possible to quantify this diffusion using a dye. The results of experiments performed with TTX (Fig. 5) also strongly suggested that any diffusion that occurred was minimal, since in these experiments the activity was maintained in the L1 and L2 ventral roots. As these were immediately contiguous to the TTX pool, if any diffusion had occurred it would have been detected due to the irreversible action of TTX. As no significant change was observed in the pattern of activity recorded in these roots, it can be concluded that the diffusion was negligible. The second point is the accuracy with which the position of the CPG was determined. Here again, the experiments performed with methylene blue showed that the inner side of the wall could be taken as a reference, since no detectable diffusion of the dye occurred beyond this point.

Figure 9 summarizes our interpretation on the organization of the spinal locomotor network. The $\mathrm{CPG}$ is located in the first two segments and sends its messages to groups of neurons distributed along the cord. From the experiments in which TTX was used, we conclude that the L1/L2 segments (Fig. 5) contain the key elements for producing a rhythmic alternating activity, since they kept their full ability to generate locomotor-like patterns when isolated. Moreover, we conclude that the lower spinal cord does not contain elements modulating the activity in $\mathrm{Ll}$ / L2. The important point here is that these two segments not only sustain the rhythm generating capabilities of the system but also contain all the elements that are needed to organize the overall locomotor pattern. Figure 8 shows that the connections between the CPG and the motoneurons are direct. The possibility cannot be ruled out, however, that part of the motoneuronal drive may be segmentally relayed, although this would involve only a minor proportion, at least in neonates in vitro. The finding that the CPG is located at the Ll/L2 level is somewhat surprising, since the segments that contain the most important pools of motoneurons are segments L.3 to L.5. They may therefore be only follower segments. This Iocation would also explain why, in many experiments, the highest level of activity recorded extracellularly in the ventral roots was always in L2.

The model that we propose in Figure 9 is clearly not compatible with previous models like the one proposed by Miller and Scott (1977). In these models, the Ia interneurons and Ren- 


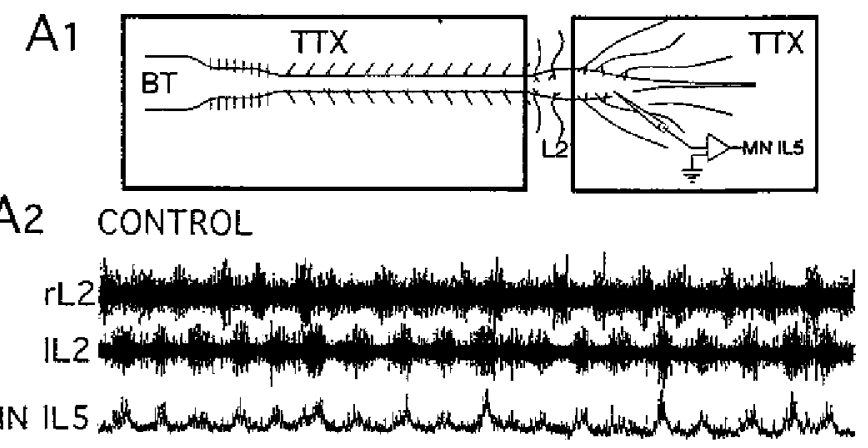

A3 TTX BT-T13/L3-sacrum

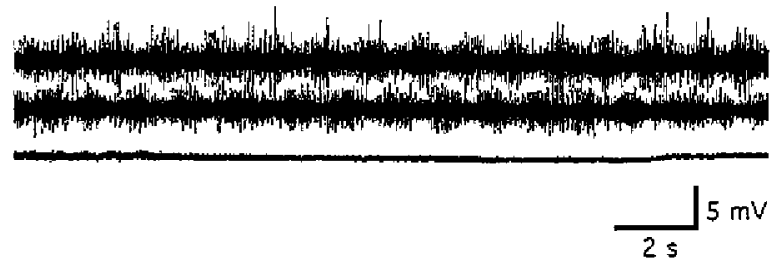

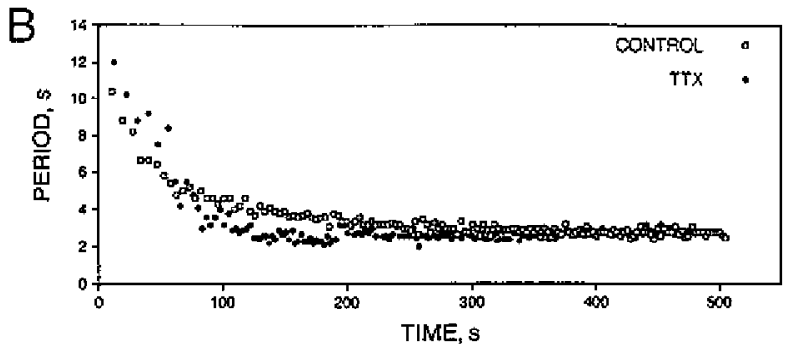
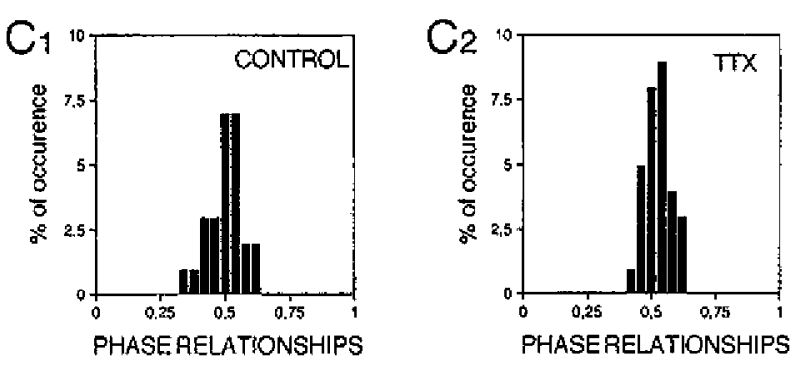

Figare 5. Persistence of bursting capabilities in TTX-isolated L1/L2 segments. Al, Diagrams of the experimental preparation. A2, Locomotor-like activity recorded in the presence of 5-HT and NMA bath applied to the L1/L2 segments. The motoneuron recorded in the left lumbar segment 5 ( $M N$ ILS) was rhythmically depolarized. A3, In the presence of TTX bath applied over the whole spinal cord except the LI/L2 segments, 5-HT/ NMA bath application still elicited locomotot-like activity. However the synaptic drive to the II 5 motoneuron was then blocked. $B$, Plot of the cycle period of locomotor-like activity as a function of time under control conditions (open square) and after TTX isolation of the L1/L2 segments The mean period value when the plateau was reached was $2.8 \pm 0.2 \mathrm{sec}$ under control conditions and $2.7 \pm 0.2$ sec in the presence of TTX; mean \pm SD. As previously reported (Sqalli Houssaini ef al., 1993), the decrease in the period value conresponded to the progressive replacement of the normal saline by the drag-containing saline until a plateau was reached. The control 5-HT/NMA bath application was followed by a wash. TTX was then bath applied for $20 \mathrm{~min}$ before a new 5-HT/NMA application, $C$. Distribution of phase relationships of right L2 bursts in the left L2 cycle. The mean values were $0.5 \pm 0.07$ under control conditions, and $0.52 \pm 0.05 \mathrm{SD}$, fl $=30$, with TTX. $A^{-C}$ are from three different experiments.

shaw cells play an important role in the generation of oscillatory behavior (for detailed review of these models, see Jordan, 1983). From our experiments in the newborn rat, it appears that these cells are not an essential element for rhythm generation since rhythm generation does not involve process at the segmental level (at which both Ia interneuron and Renshaw cells are located). In another model, it has been suggested that, in the cat, the CPG consists of a chain of paired oscillators (flexor and
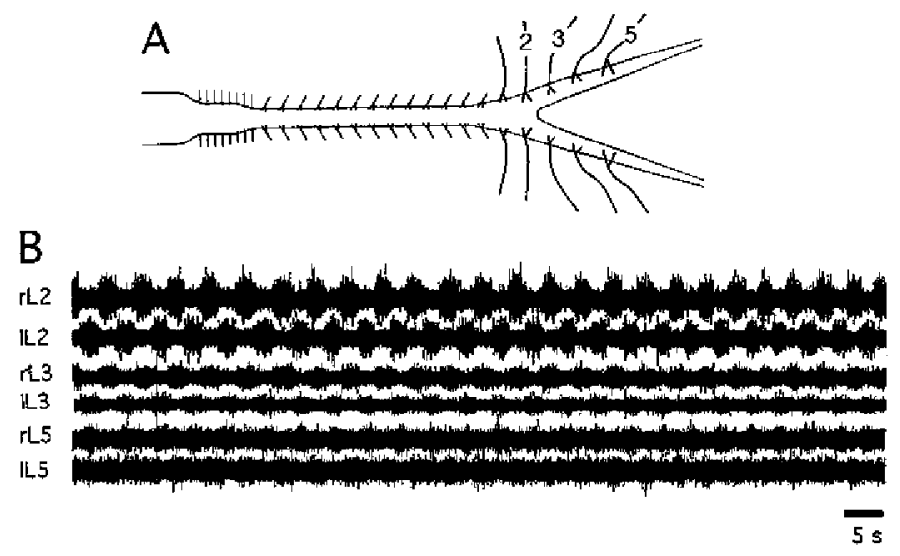

Figure 6. The right and left alternation was generated by segments L1/L2. A, Diagrams of the experimental preparation. The spinal cord was hemisected along the midline from the caudal end to $L 2$. The activity was extracellularly recorded in L2, L3, and L5. B, When 5-HT and NMA were bath applied to the spinal cord, a right and left alternating pattern was recorded in $\mathrm{L} 2$ as well as in hemisected lumbar segments L $3 / \mathrm{L} 5$. extensor), one per joint, which are coupled together (Grillner, 1985). This model is clearly compatible with the notion of localized generators, but in the present case, these joint oscillators would appear to be located at the $\mathrm{L} 1 / \mathrm{L} 2$ level rather than being distributed within the same segments as the corresponding motoneurons.

It is difficult to compare our data with results obtained in other studies using cellular labeling of the CPG, since the latter were performed on different species, that is, the rabbit (Viala et al., 1988) and the cat (Barajon et al., 1992). More recently, Kjaerulff et al. (1994), using sulforhodamine labeling of cells in the isolated neonatal rat spinal cord, have found results comparable to those previously obtained in the cat and the rabbit. In all these

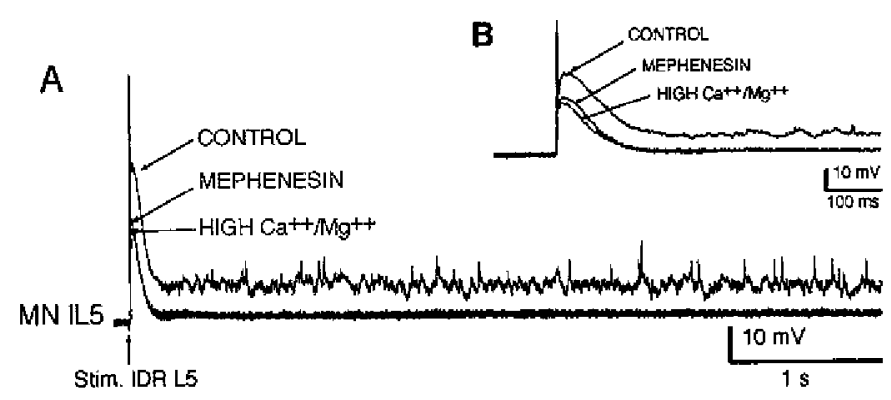

Figure 7 . Block of the polysynaptic EPSPs. A, Comparison between the responses evoked by stimulation of the dorsal root in a motoneuron under control conditions, with high $\mathrm{Ca}^{2+} / \mathrm{Mg}^{2+}$ saline and with saline containing mephenesin. $B$, The same stimulation as in $A$ but at a higher speed. Each trace is the average of 10 sweeps under each condition. 

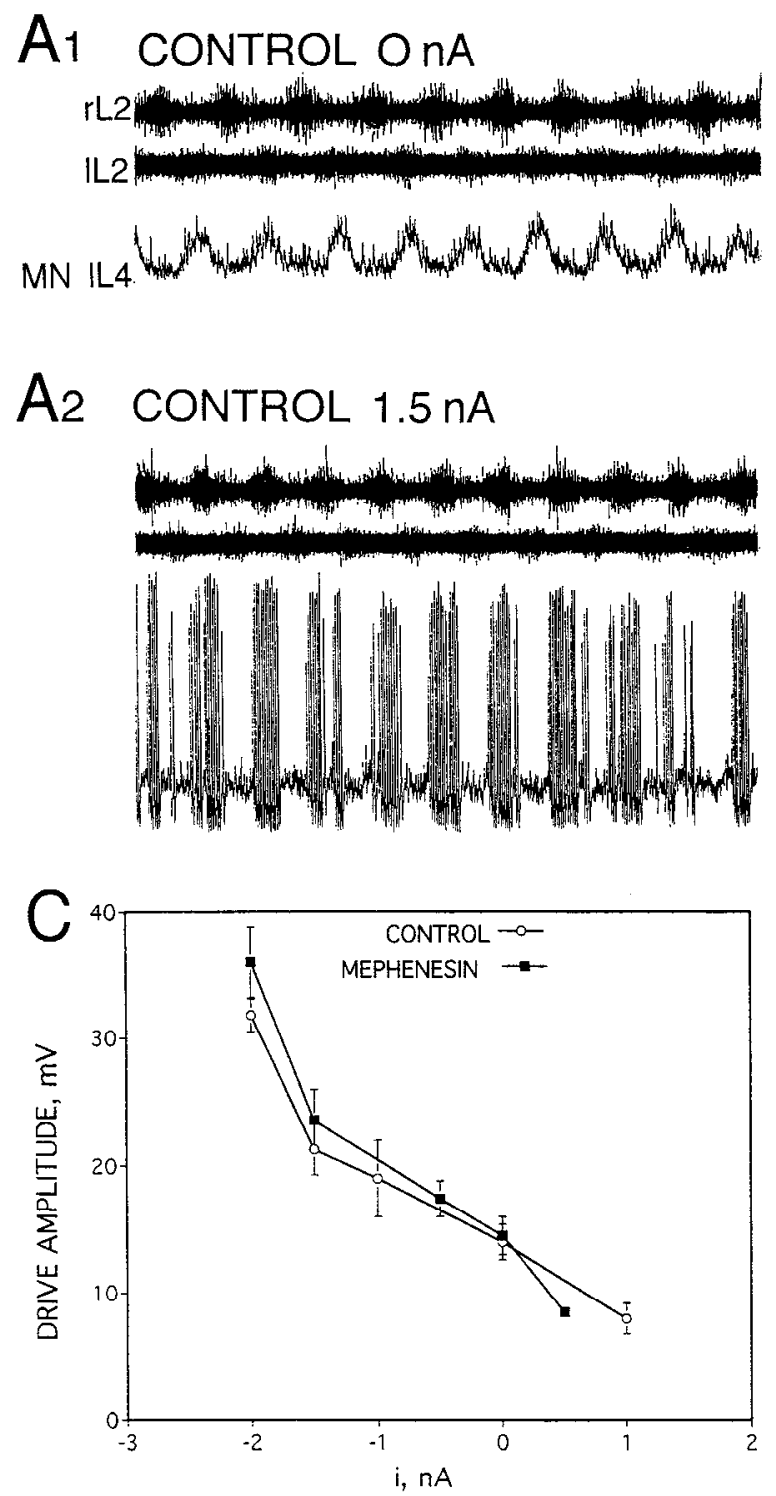
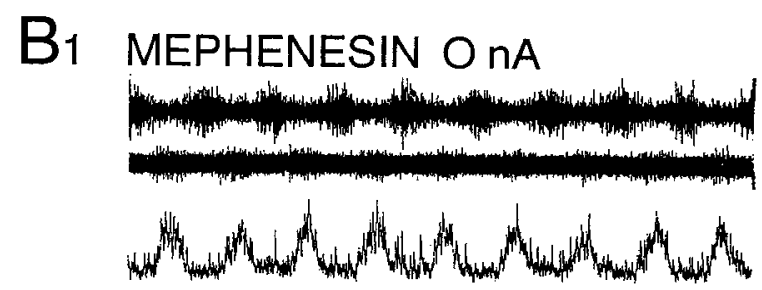

$\mathrm{B} 2$
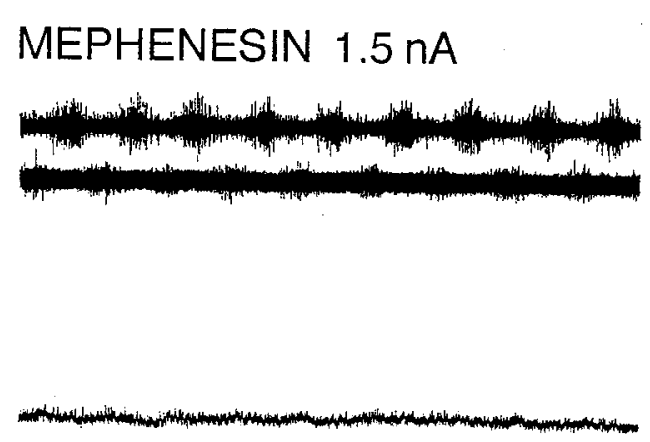

$\int_{2 \mathrm{~s}} 10 \mathrm{mV}$

Figure 8. Monosynaptic relationships between the network located in L1/L2 and the motoneurons. A, Control locomotor-like activity evoked by bath applying 5-HT/NMA to the L1/L2 segment. The motoneuron receives a rhythmic subthreshold synaptic drive (Al). In response to positive current injection $(A 2)$ the intracellularly tested motoneuron produced bursts of action potentials. $B$, In the presence of mephenesin the synaptic drive showed no change $(B I)$, but due to a decrease in its excitability, the neuron no longer discharged in response to positive current $(B 2)$. $C$, Plot of synaptic drive amplitude under control conditions (empty dots) and in saline containing mephenesin ( filled squares) during membrane depolarization or hyperpolarization with injected current. The neuron was recorded using $\mathrm{KCl}$-filled electrodes.

species, labeled (and thus active) cells were found in the intermediate gray matter and around the central canal. These methods are complementary to ours, since they make it possible to localize the relevant cells in the transverse plane. One drawback of these methods is, however, that they do not provide any information about the functional role of the labeled cells.

Several studies were previously performed in the cat with a view to physiologically locating the CPG. Grillner and Zangger (1979) established from transection experiments that the minimal spinal portion required for generating alternation in the cat was three segments ( $6 / \mathrm{L} 7 / \mathrm{S} 1$ ), although these authors were not able to conclude whether the generator was distributed throughout the spinal cord or whether it was entircly located caudally to L5. Based on experiments in which fictive scratching was analyzed when "switching-off" various parts of the spinal cord (by means of cooling) or by destroying gray matter, it was con- cluded that the bursting capacity is distributed along the spinal cord in the cat (Deliagina et al., 1983; Gelfand et al., 1988), although rostral segments had a greater capacity for generating rhythmic activity. This discrepancy with our results may, perhaps, be due to the fact that the latter authors were studying scratching activity, which is a quite different behavior from locomotor activity, as well as to differences between the species studied. Another alternative explanation is the immaturity of the system and the fact that during the postnatal period migration and changes in the location of the various elements may occur. These gross maturational processes are, however, completed at birth, as revealed by the fact that no change is observed in the location of the motoneurons from this stage to adulthood (Bennett et al., 1983). In other vertebrate species, it has also been observed that rhythm-generating network are distributed in the spinal cord (Mortin and Stein, 1989; Ho and O'Donovan, 1993). 


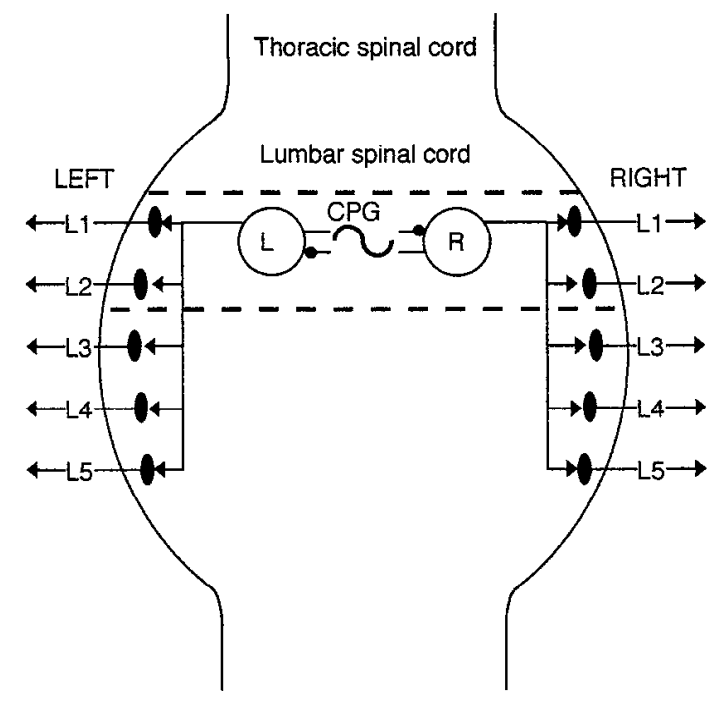

Figure 9. Diagram summarizing the spinal organization of the $\mathrm{CPG}$ for walking and its relationships with motoneurons. The spinal CPG is located at the L1/L2 segments. It produces a rhythmic alternating right and left pattern. The synaptic drive is distributed monosynaptically from this level to each of the groups of motoneurons distributed along the lumbar spinal cord.

In a detailed study on the isolated spinal cord of chick embryo, Ho and O'Donovan reported, in contrast to what we found, that the rhythmogenic capacity was distributed throughout the spinal cord, and could even be expressed in a single segment. They also found, as in the cat, that the rostral part of the lumbosacral cord expressed a greater rhythmogenic capacity. In addition, they provide evidence that separate mechanisms for rhythm and pattern generation are involved, which, for the moment, we have not been able to investigate. In contrast, arguments in favor of a localized spinal network for locomotion have been recently provided in an adult quadruped, Necturus. Wheatley et al. (1994) found that the pattern for forelimb locomotion was generated in less than two segments of the cervical spinal cord. This would confirm that our results in the newborn rat are not only a feature of an immature system.

Most of our knowledge to date about the structure of the locomotor CPG comes from studies on lower vertebrates in which it has been shown that the pattern is organized by chains of coupled oscillators. The results presented here slightly modify, however, our picture of locomotor generation. Although the output system in mammals consists of pools of motoneurons that are segmentally distributed (e.g., in the rat from LI to L6; Nicolopoulos-Stournaras and Iles, 1983), we demonstrate here that the organization at the premotoncuronal level is radically different in that the network that drives the motoneurons during locomotion lies only in the upper lumbar cord. In this context, the general organization of the locomotor CPG would be rather comparable to what has been found for mastication (Nozaki et al., 1986; see, also, review by Lund and Enomoto, 1988) or respiration (Smith et al., 1991) in which neurons generating rhythmic paltern have been found in restricted areas of the brainstem. From the practical point of view, since it is now possible to separate the rhythmic compartment from the output motoneuronal target, it becomes feasible to study the cellular properties of spinal motoneurons by modifying all the parameters in the lower segments without affecting in any way the rhythmic ac- tivity in $\mathrm{L} 1 / \mathrm{L} 2$. In addition, since the CPG network appears to be a defined entity rather than a diffusely distributed chain of coupled elements from which the rhythmic pattern arises, the possibility of a complete description at the cellular level becomes feasible.

\section{References}

Barajon I, Gossard JP, Hultborn H (1992) Induction of fos expression by activity in the spinal rhythm generator for scratching. Brain Res 588:168-172.

Bennett MR, Mc Grath PA, Davey DF, Hutchinson I (1983) Death of motoneurones during the postnatal loss of polyneuronal innervation of rat muscles. J Comp Neurol 218:351-363.

Berry MS, Pentreath VW (1976) Criteria for distinguishing between monosynaptic and polysynaptic transmission. Brain Res 105:1-20.

Cazalets JR, Grillner P, Menard I, Crémieux J, Clarac F (1990a) Two types of motor rhythms induced by NMDA and amines in an in vitro spinal cord preparation of neonatal rat. Neurosci Lett 111:116-121.

Cazalets JR, Menard I, Cremieux J, Clarac F (1990b) Variability as a characteristic of immature motor systems: an electromyographic study of swimming in the newborn rat. Behav Brain Res 40:215225.

Cazalets JR, Sqalli-Houssaini Y, Clarac F (1992) Activation of the central pattern generators for locomotion by serotonin and excitatory amino acids in neonatal rat. J Physiol (Lond) 455:187-204.

Chrachri A, Clarac F (1990) Fictive locomotion in the fourth thoracic ganglion of the crayfish, Procambarus clarlii. J Neurosci 10:707719.

Cohen AH (1987) Intersegmental coordinating system of the lamprey central pattern generator for locomotion. J Comp Physiol 160:181193.

Cohen AH, Harris-Warrick RM (1984) Strychnine eliminates alternating motor output during fictive locomotion in the lamprey. Brain Res 293:164-167.

Cohen AH, Wallén P (1978) Rhythmic locomotor activity induced in an in vitro preparation of the lamprey spinal cord. Neurosci Lett [Suppl] 1:S92.

Cohen AH, Rossignol S, Grillner S (1988) Neural control of rhythmic movements in vertebrates. New York: Wiley.

Delcomyn F (1980) Neural basis of rhythmic behavior in animals. Science 210:492-.

Deliagina, TG, Orlovsky GN, Pavlova GA (1983) The capacity for generation of rhythmic oscillations is distributed in the lumbosacral spinal cord of the cat. Exp Brain Res 53:81-90.

Gelfand IM, Orlovsky GN, Shik ML (1988) Locomotion and scratching in tetrapods. In: Neural control of rhythmic movements in vertebrates (Cohen AH, Rossignol S, Grillner S, eds), pp 167-199. New York: Wiley.

Grillner S (1985) Neurobiological bases of rhythmic motor acts in Vertebrates. Science 228:143-149.

Grillner S, Matsushima T (1991) The ncural network underlying locomotion in lamprey-synaptic and cellular mechanisms. Neuron 7:115.

Grillner S, Wallæen P (1980) Does the central pattern generator for locomotion in lamprey depend on glycine inhibition? Acta Physiol Scand 110:103-105.

Grillner S, Zangger P (1979) On the central generation of locomotion in the low spinal cat. Exp Brain Res 34:241-261.

Grillner S. McClellan A, Sigvardt K, Wallén P, Wilén M (1981) Activation of NMDA-receptors elicits "fictive locomotion" in lamprey spinal cord in vitro. Acta Physiol Scand 113:549-551.

Ho S, O'Donovan MJ (1993) Regionalization and intersegmental coordination of rhythm-generating networks in the spinal cord of the chick embryo. J Neurosci 13:1354-1371.

Jahr CE, Yoshioka K (1986) Ia afferent excitation of motoneurones in the in vitro new-born rat spinal cord is selectively antagonized by kynurenate. J Physiol (Lond) 370:515-530.

Jordan LM (1983) Factors determining motoneuron rhythmicity during fictive locomotion. Symp Soc Exp Biol 37:423-444.

Kahn JA, Roberts A (1982) The central nervous origin of the swimming motor pattern in embryos of Xenopus. J Exp Biol 99:175-184.

Kjaerulff O, Barajon I, Kiehn O (1994) Sulphorhodamine-labelled cells in the neonatal rat spinal cord following chemically induced locomotor activity in vitro. J Physiol (Lond) 478:265-273. 
Kristan SW, Calabrese RL (1976) Rhythmic swimming activity in neurones of the isolated nerve cord of the leech. Brain Res 65:613-668.

Kudo N, Yamada T (1987) N-Methyl-D,L-aspartate-induced locomotor activity in a spinal cord-hindlimb muscles preparation of the newborn rat studied in vilro. Neurosci Lell 75:43-48.

Kudo N, Ozaki S, Yamada T (1991) Ontogeny of rhythmic activity in the spinal cord of the rat. In: Neurobiological basis of human locomotion (Shimamura M, Grillner S, Edgerton VR, eds), pp 126-137. Tokyo: Japanese Science Society.

Lev-Tov A, Pinco M (1992) In vitro studies of prolonged synaptic depression in the neonatal rat spinal cord. 447:149-169.

Lund JP, Enomoto S (1988) The generation of mastication by the mammalian central nervous system. In: Neural control of rhythmic movements in vertebrates (Cohen $\mathrm{AH}$, Rossignol S, Grillner S, eds), pp 41-72. New York: Wiley.

Miller S, Scot PD (1977) The spinal locomotor generator. Exp Brain Res 30:387-403.

Mortin LI, Stein PSG (1989) Spinal cord segments containing key elements of the central pattern generator for three forms of scratch reflex in the turtle. J Neurosci 7:2285-2296.

Nozaki S, Iriki A, Nakamura Y (1986) Localization of central rhythm generator involved in cortically induced rhythmical masticatoty jawopening movement in the guinea pig. J Neurophysiol 55:806-825.

Roberts A, Soffe SR, Clarke JDW, Dale N (1983) Initiation and control of swimming in amphibian embryos. Symp Soc Exp Biol 37:261284.
Sillar KT, Skorupski P (1986) Central input to primary afferent neuron in crayfish Pacifastacus lenisculus is correlated with rhythmic motor output thoracic ganglia. J Neurophysiol 55:678-689.

Soffe SR (1987) Ionic and pharmacological properties of reciprocal inhibition in Xenopus embryo motoneurones. J Physiol (Lond) 382: 463-473.

Smith JC, Feldman JL Schmidt BJ (1988) Neural mechanisms generating locomotion studied in mammalian brain stem-spinal cord in vitro. FASEB 2:2283-2288.

Smith JC, Ellenberger HH, Ballanyi K, Richter DW, Feldman JL (1991) Pre-Bötzinger complex: a brainstem region that may generate respiratory rhythm in mammals. Science 254:726-729.

Sqalli-Houssaini Y, Cazalets JR, Clarac F (1991) A cooling/heating system for use with in vitro preparations: study of temperature effects on newborn rat rhythmic activities. J Neurosci Methods 39:131-139.

Sqalli-Houssaini Y, Cazalets JR, Clarac F (1993) Oscillatory properties of the central pattern generator for locomotion in neonatal rats. $J$ Neurophysiol 70:803-813.

Tunstall MJ, Sillar KT (1993) Physiological and developmental aspects of intersegmental coordination in Xenopus embryos and tadpoles. Semin Neurosci 5:29-40.

Viala D, Buisseret Delmas C, Portal JJ (1988) $\Lambda \mathrm{n}$ attempt to localize the lumbar locomotor generator in the rabbit using 2-deoxy- $\left({ }^{14} \mathrm{C}\right)$ glucose autoradiography. Neurosci Lett 86:139-143.

Wheatley M, Jovanovic K, Stein RB, Lawson V (1994) The activity of interneurons during locomotion in the in vitro Necturus spinal cord. J Neurophysiol 71:2025-2032. 